\title{
Motivación escolar, inteligencia emocional y rendimiento académico en estudiantes de educación secundaria obligatoria
}

\author{
School Motivation, Emotional Intelligence and Academic \\ Performance in Students of Secondary Education
}

\author{
Pablo Usán Supervía ${ }^{1}$ \\ Carlos Salavera Bordás ${ }^{2}$ \\ Universidad de Zaragoza, España
}

Resumen. El objetivo del presente trabajo fue analizar la relación entre la motivación escolar, la inteligencia emocional y el rendimiento académico en una muestra de 3512 estudiantes adolescentes pertenecientes a 18 centros educativos. Los instrumentos utilizados fueron la Escala de Motivación Educativa (EME-S), la Traid MetaMood Scale-24 (TMMS-24) y el rendimiento académico se cuantificó a través de la nota media de los alumnos. Los resultados mostraron relaciones significativas entre motivaciones escolares intrínsecas e inteligencia emocional, en mayor medida que con las extrínsecas y amotivación. Asimismo, la regulación emocional, la motivación intrínseca hacia el conocimiento y la amotivación predijeron el rendimiento escolar de los alumnos. Se desprende la influencia de las tres variables en el desarrollo personal y académico de los estudiantes.

Palabras clave. Motivación escolar, inteligencia emocional, rendimiento académico, estudiantes, adolescentes.

Abstract. The aim of this study was to analyze the relationship between school motivation, emotional intelligence and academic performance in a sample of 3512 adolescent students belonging to 18 educational centers. The instruments used were the spanish versions of the Educational Motivation Scale (EME-S), the Traid Meta-Mood Scale-24 (TMMS-24) and the academic performance was quantified through the average grade of the students. The results showed stronger relationships between intrinsic school motivations and emotional intelligence than extrinsic motivations and amotivation. Likewise, emotional regulation, intrinsic motivation and demotivation predicted students' school performance. The influence of school motivation, emotional intelligence and academic performance in the personal and academic development of the students.

Keywords. School motivation, emotional intelligence, academic performance, students, teenagers.

${ }^{1}$ Pablo Usán Supervía. Universidad de Zaragoza, España. Dirección Postal: Facultad de Ciencias Humanas y de la Educación (Huesca) C/Valentín Carderera n4, 22003. E-mail: pablousan@hotmail.com

${ }^{2}$ Carlos Salavera Bordás.Universidad de Zaragoza, España. E-mail: salavera@unizar.es

\section{$@ \oplus \Theta \Theta$}




\section{Introducción}

Durante la escolaridad de los alumnos en los centros educativos se producen numerosas situaciones, personales y contextuales, que pueden afectar de manera significativa a su proceso de formación, lo cual resulta especialmente relevante en la etapa de secundaria. Esta, coincidiendo con el periodo de la adolescencia, se revela como una fase clave en la vida de una persona, previo paso a la edad adulta, donde se producen los mayores cambios físicos y psicológicos que forjarán la personalidad adulta del estudiante (Gómez-Fraguela, Fernández, Romero, \& Luengo, 2008).

De este modo, ciertos alumnos durante su etapa escolar, pueden no disponer o hacer uso de las estrategias y competencias necesarias para solventar con éxito las exigencias y demandas de la vida académica, y pueden llegar a experimentar actitudes negativas hacia ella, pérdida de interés en sus estudios o, incluso, agotamiento físico y psíquico (Palacio, Caballero, González, Gravini, \& Contreras, 2012). Todas estas experiencias pueden desembocar en una falta de motivación absoluta del alumnado y mermar su rendimiento e, incluso, conducir al abandono prematuro de sus estudios (Musitu, Jiménez, \& Murgui, 2012).

$\mathrm{Al}$ hacer referencia a las explicaciones o causas del bajo rendimiento o al fracaso escolar, el concepto de motivación aparece de manera recurrente. Los modelos motivacionales consideran a la motivación como un ente que explica el inicio, dirección y perseverancia de una conducta hacia una determinada meta académica, centrada en cuestiones inherentes al propio proceso de aprendizaje: el rendimiento académico, la valoración social o, incluso, la evitación del trabajo (Alemany, Campoy, Ortiz, \& Benzaquén, 2015). De este modo, Järvenoja (2010) sugiere que la motivación resulta un proceso activo y dinámico por el cual una persona se mantiene en una tarea con el fin de conseguir sus propósitos. Así, para el logro de los objetivos del alumnado, no solo se requieren capacidades normativas y conocimientos específicos en una materia sino, también, la disposición y motivación para ello (Núñez, 2009).

De esta manera y centrada en el contexto académico, se entiende por motivación escolar el conjunto de creencias que el alumnado posee con respecto a sus objetivos y fines, revelando el por qué una meta es importante para él y deduciendo una explicación acerca de la persistencia en su conducta (Rosário \& Högemann, 2015). Además, el interés y la persistencia son elementos de la voluntad que mantienen la motivación e inciden en la concentración y disposición en la tarea del alumnado (Gaeta \& Cavazos, 2015).

De esta manera, la teoría de la autodeterminación (TAD) (Deci \& Ryan, 2002) actúa como un continuo que abarca diferentes grados de autodeterminación en la conducta, desde la no autodeterminada hasta la más autodeterminada (Moreno \& Martínez, 2006). Durante todo este recorrido, se abarcan tres tipos fundamentales de motivación que cuentan con su propia estructura y se encuentran reguladas por el sujeto de manera interna o externa: la motivación intrínseca, la motivación extrínseca y la amotivación o, lo que es lo mismo, la falta de motivación.

La motivación intrínseca hace referencia al desarrollo de una actividad por la satisfacción inherente derivada de ella, no precisa de reforzamientos externos y resulta en un constructo multidimensional en el que se distinguen tres tipos. El primero es la motivación intrínseca 
hacia las experiencias estimulantes, cuando un sujeto se involucra en una actividad para divertirse o experimentar sensaciones estimulantes y positivas derivadas de la propia dedicación a la actividad; motivación intrínseca hacia el conocimiento, relacionada con el deseo por el aprendizaje de nuevos conceptos y, por último, la motivación intrínseca hacia el logro, caracterizada por el afán de superación o culminación de objetivos personales propuestos.

En la motivación extrínseca, la conducta adquiere significado porque está dirigida hacia un fin y no por sí misma. En ella se distinguen tres tipos de motivaciones, ordenadas de menor a mayor nivel de autodeterminación: la motivación extrínseca externa, que refiere a las recompensas o evitación de castigos en la realización de una actividad; la motivación extrínseca identificada, donde el sujeto atribuye un valor personal a su conducta y resulta percibida como una elección del propio individuo porque la considera adecuada e importante. Finalmente, la motivación extrínseca introyectada, en la que el sujeto lleva a cabo su actividad para evitar su culpa o realzar su ego en el desempeño de ella.

Por último, la amotivación, resulta un estado de falta de motivación en la realización de la tarea. Implica una escasa valoración de ella, ausencia de control en la conducta o, incluso, percepción de incompetencia e inoperancia para llevarla a cabo de manera satisfactoria.

Muchos son los estudios que relacionan, en población adolescente, conceptos asociados a una adecuada motivación escolar como su estrecha relación con el engagement académico (Usán, Salavera, \& Domper, 2018). Por ejemplo, percepción de autoeficacia en el desarrollo de tareas escolares (Bresó, Schaufeli, \& Salanova, 2011), inteligencia emocional (IE) (Salavera, Usán, \& Jarie, 2017), bajo índice de abandono escolar (Musitu et al., 2012) así como, en términos generales, mayor felicidad académica de los estudiantes y ausencia de consumo de drogas en adolescentes (Usán \& Salavera, 2017).

Por otro lado, la inteligencia emocional se conoce como la capacidad de una persona para procesar la información que le proporcionan las emociones a su alrededor (Mayer, Salovey, \& Caruso, 2000). Las personas emocionalmente inteligentes son aquellas capaces de atender a las emociones percibidas en su contexto más próximo, comprendiendo sus posibles causas y consecuencias y desarrollando, así, las estrategias necesarias para regular o manejar los diferentes estados emocionales (Mayer \& Salovey, 1997; Mayer, Salovey, \& Caruso, 2000; Pena \& Extremera, 2012).

La literatura científica en torno al estudio de la inteligencia emocional en el ámbito educativo resulta amplia y se enfoca en diferentes poblaciones y contextos. La mayoría de los estudios se han centrado en investigar su incidencia en el bienestar de los estudiantes en Educación Primaria (Salavera, Usán, \& Jarie, 2018), en Educación Secundaria Obligatoria (ESO) (Extremera, Durán, \& Rey, 2009), estudiantes universitarios (Salavera, Usán, Jarie, \& Lucha, 2018) o, incluso, en docentes (Andaur \& Berger, 2018). Lo anterior denota la estrecha relación de la inteligencia con el bienestar subjetivo, satisfacción vital y felicidad académica de los estudiantes.

En la actualidad, los términos de motivación e inteligencia emocional son empleados indistintamente en el ámbito educativo. Se considera la inteligencia emocional como una manera de interactuar con todo aquello que rodea al individuo quien tiene muy 
en cuenta la motivación (Goleman, 2012), la cual lleva a considerar las emociones como un aspecto integral de la motivación en la medida en que esta desencadena la conducta orientada a un fin (Usán, Salavera, Merino, \& Jarie, 2018). En consecuencia, tal y como apunta Anaya (2012), los sistemas motivacionales y de inteligencia emocional interactúan y se sostienen mutuamente al servicio de las metas perseguidas, en detrimento de posicionamientos interdependientes (Sanz, Menéndez, Rivero, \& Conde, 2009). De este modo, las emociones suponen un papel importante en la adaptación de los estudiantes en su centro escolar en cuanto al manejo emocional de todas aquellas variables contextuales y personales que se producen a lo largo del curso académico y que pueden determinar cuestiones tan importantes como el bienestar personal, la motivación escolar o el rendimiento académico, entre otros.

Por último, el rendimiento académico es una de las dimensiones más importantes en el proceso de enseñanza-aprendizaje (Garzón, Pinzón, Del Riesgo, Rojas, \& Salamanca, 2010), el cual es aceptado como un concepto multidimensional, amplio y relativo en función de los diversos objetivos y resultados esperados en la acción educativa (Abalde, Barca, Muñoz, \& Fernando, 2009). Otros estudios lo consideran como la medición de la efectividad escolar o logros académicos (Niebla \& Hernández, 2007; Tilano, Henao, \& Restrepo, 2009). A pesar de que las calificaciones numéricas son uno de los factores más empleados en la literatura científica y vaticinadores de mayor estabilidad que se conocen en relación con el rendimiento académico del alumnado (Barca, Peralbo, Porto, Marcos, \& Brenlla, 2011), se han utilizado diferentes formas de cuantificar el rendimiento como pruebas estandarizadas (Carmona, Sánchez, \& Bakieva, 2011), el número de suspensos (Díaz, 2003), los cursos escolares repetidos (Hernando, Oliva, \& Pertegal, 2012), cantidad de tiempo dedicado al estudio (Molleda, \& Herrero, 2009) o, incluso, la combinación de varios de ellos (Rosário et al., 2012).

De esta manera, interrelacionando las variables de motivación escolar y rendimiento académico, la literatura científica deja varios estudios al respecto. Algunos de ellos consideran que las motivaciones intrínsecas se relacionan con un mayor rendimiento académico, así como con otros mediadores motivacionales cognitivos y conductuales más adaptativos dentro del proceso de aprendizaje (Zimmerman, 2008). Otros, aluden a la posesión predominante de motivaciones intrínsecas en detrimento de extrínsecas en alumnado de rendimiento bajo, aunque no resulta un factor predictor de este (Barca et al., 2011; Pintrich \& Schunk, 2006).

Navas y Sampascual (2008) aluden a que los estudiantes de alto rendimiento se diferencian de los de bajo en que sus niveles de motivación intrínseca y orientaciones de meta hacia la tarea resultan más elevados en los primeros que las orientadas extrínsecamente. Sin embargo, el nivel predictivo de las motivaciones intrínsecas para explicar el rendimiento académico no ha sido esclarecedor y los resultados encontrados en las investigaciones son diversos e, incluso, en una dirección opuesta prevaleciendo las motivaciones extrínsecas (Anderman, Anderman, Yough, \& Gimbert, 2010).

Por otra parte, existen estudios que afirman que elevados índices de motivación intrínseca y ausencia de amotivación se relacionan positivamente con altos niveles de rendimiento académico; mientras que, desde una perspectiva contraria, las motivaciones extrínsecas 
y mayores niveles de amotivación se relacionarían con la orientación al ego pero no necesariamente con un bajo rendimiento académico y otras conductas menos adaptativas (Aleman, Trías, \& Curione, 2011; Salavera \& Usán, 2018). Además, Miñano y Castejón (2011) aluden a la importancia de la modulación de las experiencias de éxito/fracaso en la vida académica de los estudiantes para definir sus motivaciones a la hora de enfrentarse a las tareas y quehaceres académicos.

Ahora bien, relacionando las variables de inteligencia emocional y rendimiento académico, la literatura científica ofrece ciertos estudios que relacionan ambas en población escolar adolescente con resultados contrapuestos. Por un lado, diversas investigaciones denotan la relación entre inteligencia emocional y rendimiento académico. Vallejo, Martínez, García y Rodríguez (2012) concluyen que el rendimiento se encuentra influido por la inteligencia emocional denotando, además, diferencias entre estudiantes con baja inteligencia emocional y los alumnos con alta; no se observan diferencias entre los de inteligencia emocional adecuada o media con los otros dos.

Por otro lado, diversas investigaciones muestran una línea contraria a los trabajos anteriormente citados. Jiménez y López-Zafra (2009), en un estudio sobre la incidencia de las emociones en el contexto educativo, afirman que los resultados se muestran inconsistentes y no definitorios, proponiendo nuevas metodologías de estudio. Hernández (2005) niega la capacidad predictiva de la IE sobre el rendimiento académico en adolescentes escolares. Martínez (2010) no encuentra relaciones significativas entre la inteligencia emocional y el rendimiento académico; indica, además, que aquellos estudiantes con mayor nivel de este tienden a obtener menores niveles de inteligencia emocional y viceversa.

Por todo lo anterior, y siguiendo a Cera, Almagro, Conde y Sáenz-López (2015), se disponen de pocos trabajos de investigación que aborden de manera específica los vínculos existentes entre la motivación escolar, la inteligencia emocional y el rendimiento académico en el contexto educativo resultando necesarios más estudios que indaguen y amplíen la comprensión y conocimiento de la interrelación entre los diferentes constructos enunciados en aras del desarrollo personal y académico de los alumnos en los centros educativos. De este modo, el objetivo del estudio se centra en analizar la relación entre la motivación escolar, las orientaciones de meta y la inteligencia emocional en una muestra de adolescentes escolares.

De esta manera, y con base en el objetivo anteriormente enunciado, se postulan tres hipótesis: (a) Aquellos estudiantes con unos elevados índices de motivaciones intrínsecas se relacionarán positivamente la inteligencia emocional y el rendimiento académico, siguiendo una línea de conductas más adaptativas que, (b) Aquellos estudiantes que obtengan mayores niveles de motivación extrínseca, relacionados positivamente con la amotivación $y$, de manera negativa, con las dimensiones de la inteligencia emocional y el rendimiento académico, en una línea de conductas menos adaptativas. Además, (c) Las motivaciones intrínsecas y la inteligencia emocional predecirán positivamente el rendimiento académico mientras que, las motivaciones extrínsecas y amotivación, predecirán el rendimiento académico de manera negativa. 


\section{Método}

\section{Muestra}

El muestreo realizado fue de tipo estratificado, donde la unidad primaria es la titularidad de los centros y la etapa secundaria el nivel educativo ( $\left({ }^{\circ} \mathrm{Eso}-4^{\circ} \mathrm{Eso}\right)$. La muestra estuvo formada por 3512 estudiantes; de ellos, el género masculino fue $N=1816$ (51.07\%) y femenino $N=1696(48.29 \%)$. Estos pertenecen a 18 centros públicos de Educación Secundaria Obligatoria (ESO) de la provincia de Zaragoza, con edades comprendidas entre los 12 y 18 años $(M=14.55 ; D T=1.68)$, quienes participaron de manera voluntaria en el estudio, el cual se rigió en todo momento por las consideraciones éticas de la Declaración de Helsinki (Asociación Médica Mundial, 2000).

El estudio también cumplió los criterios éticos de investigación con seres humanos (consentimiento informado de padres y madres, profesores y estudiantes y derecho de información, protección de datos personales y garantías de confidencialidad, no discriminación, gratuidad y posibilidad de abandonar el estudio en cualquiera de sus fases). Realizado el cálculo de la representatividad de la muestra, con un nivel de confianza del $99 \%$ y un error muestral del 5\%, se obtuvo que la muestra final encuestada resultó representativa de la provincia de Zaragoza. El trabajo se diseñó como un estudio retrospectivo expostfacto (Montero \& León, 2007).

Tabla 1

\begin{tabular}{|c|c|c|c|}
\hline & & $f$ & $\%$ \\
\hline \multirow{7}{*}{ Edad } & 12 años & 307 & 17.89 \\
\hline & 13 años & 293 & 16.54 \\
\hline & 14 años & 403 & 24.02 \\
\hline & 15 años & 417 & 23.93 \\
\hline & 16 años & 269 & 14.03 \\
\hline & 17 años & 56 & 2.71 \\
\hline & 18 años & 11 & 0.88 \\
\hline \multirow{4}{*}{ Curso académico } & $1^{\circ} \mathrm{ESO}$ & 338 & 20.09 \\
\hline & $2^{\circ} \mathrm{ESO}$ & 436 & 25.63 \\
\hline & $3^{\circ} \mathrm{ESO}$ & 567 & 32.11 \\
\hline & $4^{\circ} \mathrm{ESO}$ & 415 & 22.17 \\
\hline
\end{tabular}

\section{Instrumentos}

Con la finalidad de percibir la motivación escolar de los estudiantes se utilizó la Escala de Motivación Educativa (EME-S) adaptada al contexto de educación secundaria (Núñez, Martín-Albó, Navarro, \& Suárez, 2010). Se compone de 28 ítems distribuidos en tres grandes dimensiones con siete subescalas totales de 4 ítems que responden a las razones o motivos por las cuáles los estudiantes van a la escuela; para cada uno, se presentará un 
ejemplo: motivación intrínseca a experiencias estimulantes $(\alpha=.79)$ : "Porque, para mí, el instituto es divertido"; al conocimiento $(\alpha=.86)$ : "Porque siento placer y satisfacción cuando aprendo cosas nuevas"; y al logro $(\alpha=.84)$ : "Por la satisfacción que siento cuando voy superando actividades académicas difíciles"; motivación extrínseca externa $(\alpha=.83)$ : "Para conseguir un puesto de trabajo más prestigioso", identificada $(\alpha=.84)$ : "Porque me permitirá acceder al mercado laboral en el campo que más me gusta" e introyectada ( $\alpha=$ .82): "Porque cuando realizo bien las tareas en clase me siento importante" y, por último, amotivación $(\alpha=.85)$ : "No lo sé, no entiendo qué hago en el instituto". Las respuestas se erigen en una escala tipo Likert que oscila desde "Muy en desacuerdo" (1) hasta "Muy de acuerdo" (5). La fiabilidad del instrumento original denota un alfa de Cronbach de .80; mientras que, en esta investigación, otorgó una significancia de .82.

Con la finalidad de percibir la inteligencia emocional de los estudiantes se utilizó la escala Traid Meta-Mood Scale-24 (TMMS-24) (Salovey, Mayer, Goldman, Turvey, \& Palfai, 1995) en una versión reducida y adaptada a la población española por Fernández-Berrocal, Extremera y Ramos (2004). La escala contiene tres dimensiones con 8 ítems cada una de ellas: atención emocional (5) $(\alpha=.79)$ : "Presto mucha atención a mis sentimientos"; claridad de sentimientos (5) ( $\alpha=.83)$ : "Frecuentemente puedo definir mis sentimientos" y reparación emocional $(5)(\alpha=.82)$ : "Aunque me sienta mal, procuro pensar cosas agradables". Las respuestas se disponen en una escala tipo Likert que oscila desde "Muy en desacuerdo" (1) hasta "Muy de acuerdo" (5). La fiabilidad del instrumento original describe un alfa de Cronbach de .83; mientras que, en nuestra investigación, otorgó una significancia de .84 .

Por último, con la finalidad de conocer el rendimiento académico de los estudiantes, se obtuvo la nota media (expresada por un número entero y dos decimales) de todas las asignaturas del último trimestre del curso, momento temporal en el que se suministraron las pruebas. Este es uno de los procedimientos más empleados y vaticinadores de mayor estabilidad en relación con el rendimiento académico del alumnado (Barca et al., 2011; Córdoba, García, Luengo, Vizuete, \& Feu, 2012). La fiabilidad de dicha variable denota un $\alpha$ de .84.

\section{Procedimiento}

En la realización del estudio se contó con la aprobación de los diferentes centros educativos de secundaria, así como de los padres/tutores del alumnado por medio de consentimiento informado para poder participar en la investigación. Un día de la semana, acordado previamente con jefatura de estudios, se llevó a cabo la cumplimentación de los cuestionarios en cada una de las clases del mismo centro. Todos los sujetos y sus padres/tutores fueron previamente informados de la naturaleza del estudio, participaron de manera voluntaria, respetando, de este modo, las directrices éticas de la Declaración de Helsinki (Asociación Médica Mundial, AMM, 2000) en todos sus términos.

\section{Análisis de datos}

Se procedió a realizar una estadística descriptiva con la finalidad de conocer los datos sociodemográficos de la muestra, así como las diferentes variadas estudiadas. Posteriormente, se llevaron a cabo correlaciones entre las variables de motivación escolar, 
inteligencia emocional y rendimiento académico, procesadas y analizadas mediante el programa estadístico IBM SPSS v22.0. A su vez, para estimar la predicción de las diferentes motivaciones y la inteligencia emocional sobre el rendimiento académico, se realizó un análisis de regresión múltiple por pasos. Para todas las operaciones, se tuvo en cuenta un nivel de significación $p<.05$, y se trabajó con un nivel de confianza del 95\%.

\section{Resultados}

Análisis correlacional entre motivación escolar, inteligencia emocional y rendimiento académico

Analizando las variables de motivación escolar, inteligencia emocional y rendimiento académico, se aprecian correlaciones significativas entre varias de ellas (véase tabla 2). De esta manera, analizando la variable de motivación escolar, se observaron correlaciones significativas entre las seis primeras (motivación intrínseca hacia las experiencias estimulantes, el conocimiento y al logro y motivación extrínseca externa, identificada e introyectada), en donde se dieron índices más elevados entre las tres primeras motivaciones intrínsecas y en donde se encontró la mayor correlación entre la motivación hacia el conocimiento y la motivación hacia el logro $(r=.717, p<.01)$ La amotivación presentó una relación inversa con estas tres últimas y de manera positiva con la motivación extrínseca externa $(r=.257, p<.01)$.

Con respecto a la inteligencia emocional, se relacionó positivamente con la mayoría de motivaciones intrínsecas y extrínsecas, especialmente en las dimensiones de atención y regulación emocional, donde se hallaron índices más elevados. La amotivación, correlacionó de forma negativa con la atención emocional $(r=.320, p<.01)$, la claridad emocional $(r=$ $.271, p<.01)$ y con la regulación emocional $(r=.094, p<.05)$.

Por último, el rendimiento académico correlacionó positivamente con todas las dimensiones de la motivación intrínseca y extrínseca, con índices más acusadas en las primeras, del mismo modo que con todas las dimensiones que componen la inteligencia emocional. A su vez, amotivación y rendimiento escolar correlacionaron negativamente $(r=-.193, p<.0 .1)$.

\section{Predicción de la motivación escolar y la inteligencia emocional sobre el rendimiento académico}

Para especificar el valor predictivo de las motivaciones intrínsecas y extrínsecas, así como de la inteligencia emocional sobre el rendimiento académico, se realizó una regresión múltiple por pasos, seleccionando las dimensiones de ambas variables como variables predictoras al mismo tiempo que el rendimiento académico como variable de criterio. La tabla 3 muestra el paso final a la hora de introducir las variables que fueron significativas, prediciendo la probabilidad de su influencia sobre el rendimiento académico. Como se puede observar, el modelo mostró como variables predictoras la motivación intrínseca hacia el conocimiento, la regulación emocional y la amotivación o falta de motivación (esta última de forma negativa), las cuales formaron parte de la ecuación.

\section{Discusión}

El objetivo del estudio fue analizar la relación entre la motivación escolar, la inteligencia emocional y el rendimiento académico en una muestra de estudiantes adolescentes en la etapa de Educación Secundaria Obligatoria. 


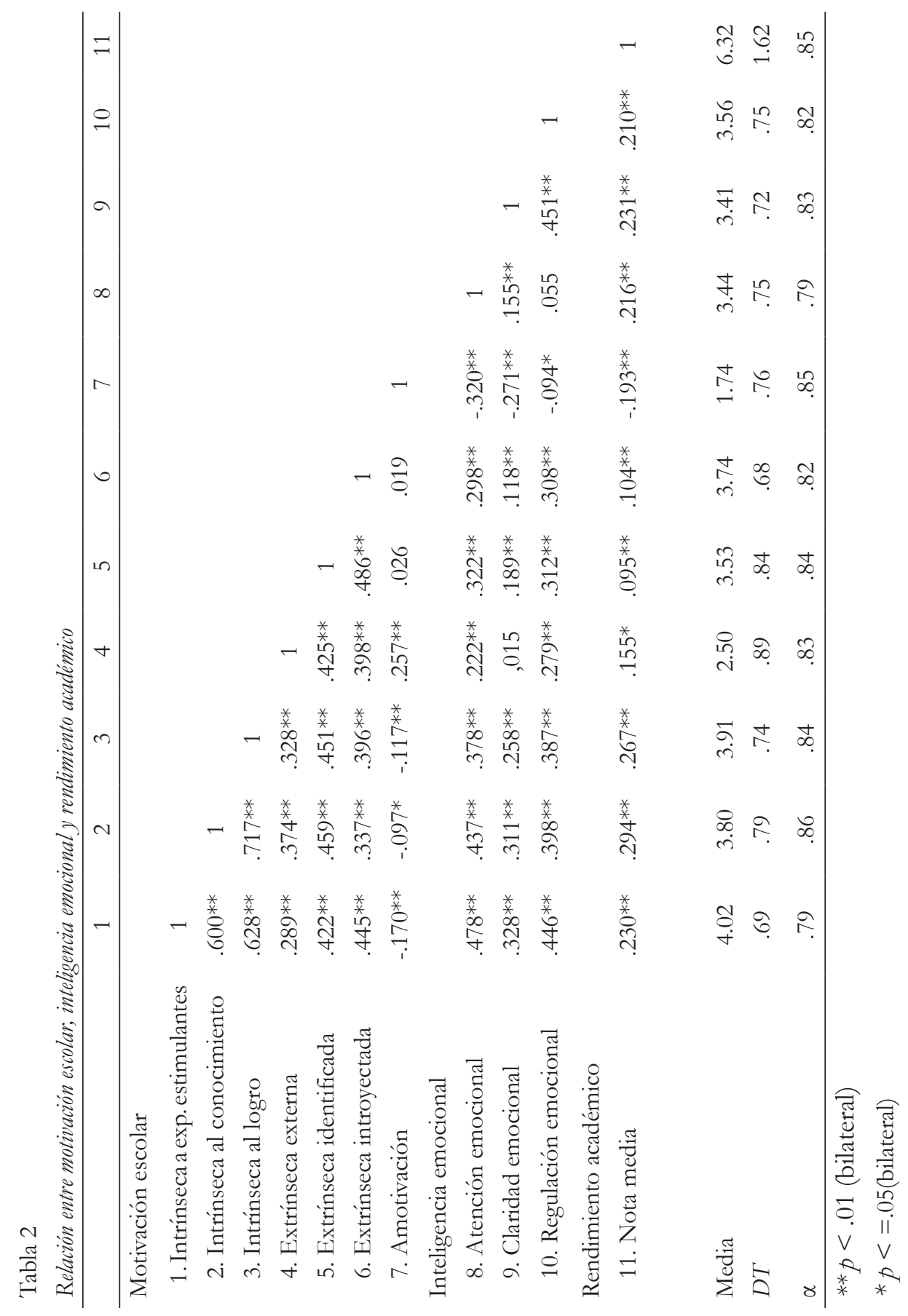

Actualidades en Psicología, 32(125), 2018, 95-112 
Tabla 3

Motivación escolar e inteligencia emocional como predictores del rendimiento académico

\begin{tabular}{lccccc}
\hline \multicolumn{1}{c}{ Último paso } & $\beta$ & s.e. & $R^{2}$ & $t$ & $p$ \\
\hline (Constante) & 2.898 & .194 & & 14.971 & .001 \\
Motivación Intrínseca-Conocimiento & .210 & .041 & .320 & 4.673 & .001 \\
Inteligencia emocional-Regulación emocional & .172 & .040 & .335 & 3.827 & .001 \\
Amotivación & -.156 & .039 & .349 & -3.772 & .001 \\
\hline
\end{tabular}

La primera hipótesis que se postuló fue, precisamente, que aquellos estudiantes con altos índices en motivaciones intrínsecas se relacionarían positivamente con la inteligencia emocional y el rendimiento académico, siguiendo una línea de conductas adaptativas. Dicha hipótesis se cumplió en su totalidad; los resultados de la investigación revelaron la estrecha relación entre dichas variables. De este modo, aquellos estudiantes más motivados intrínsecamente hacia experiencias estimulantes, el conocimiento y el logro se relacionaron con mayores índices en atención, claridad y regulación emocional del mismo modo que con el rendimiento académico.

La literatura científica suele coincidir con los resultados hallados en esta investigación. De este modo, Pérez y Castejón (2007) encuentran relaciones positivas entre motivaciones predominantemente intrínsecas y las tres dimensiones que componen la inteligencia emocional, unidas a una mayor dedicación en la tarea y divertimento escolar. Skinner, Furrer, Marchand y Kinderman (2008), en una muestra de estudiantes de escuelas primarias y secundarias, encuentran relaciones entre estas dos variables unidas a la adhesión y compromiso de los estudiantes con la institución escolar.

Ferriz, Sicilia y Sáenz (2013) destacaron relaciones entre los alumnos más motivados intrínsecamente y sus niveles de inteligencia emocional, unido a un mayor rendimiento académico. De este modo, se evidenció una línea de conductas adaptativas entre las variables de motivación intrínseca, inteligencia emocional y rendimiento académico que, en definitiva, conducen a un mayor bienestar psicológico y emocional de los estudiantes adolescentes (Gaeta et al., 2015).

La segunda hipótesis de la investigación aludía a una posible relación positiva de la motivación extrínseca con la amotivación y, de manera negativa, con las dimensiones de la inteligencia emocional y el rendimiento académico, en una línea de conductas menos adaptativas. La hipótesis descrita se cumplió de manera parcial; los resultados de la investigación describieron la significación estadística de las motivaciones extrínsecas con las demás variables citadas, pero no se encontraron relaciones entre las motivaciones extrínsecas y la falta de motivación (salvo escasa correlación con la motivación extrínseca). Tampoco se hallaron relaciones con las dimensiones de la inteligencia emocional y rendimiento académico de manera negativa como se había pronosticado, sino a la inversa: se hallaron significaciones positivas, especialmente con la atención y regulación emocional, dimensiones de la inteligencia emocional.

Con todo ello, se desprende el hecho de que la asunción de unas motivaciones predominantemente extrínsecas no tiene por qué estar vinculada con la falta de motivación 
escolar o inteligencia emocional de igual forma que un rendimiento académico menor, como se ha visto reflejado en estos resultados. Tsouloupas, Carson, Matthews, Grawitch y Barber (2010) sostienen las relaciones entre motivaciones extrínsecas y rendimiento académico pero no como un factor influyente a la hora de conducir a un rendimiento académico menor. Por último, Delgado, Inglés, García-Fernández, Castejón y Valle (2010) aluden a otras variables de peso, como la edad o el género, para determinar el rendimiento académico en adolescentes en vez de sus motivaciones extrínsecas.

Siguiendo con estos estudios, las variables descritas no actuarían contradictoriamente de manera dicotómica entre sí, sino que formarían parte de un mismo patrón de conducta, el cual denotaría que aquellos alumnos con un mayor rendimiento escolar no tienen por qué estar asociados, únicamente, a motivaciones predominantemente intrínsecas. Esto puede ser debido a que ciertos estudiantes pueden estar motivados intrínseca y extrínsecamente a la vez, haciendo uso de sus niveles según las demandas de la tarea y/o variables personales, actuando de manera ortogonal (Alexandris, 2013). Tal y como apuntan Saies, Arribas, Cecchini, De Cos y Otaegi (2014), la motivación extrínseca no siempre lleva asociados comportamientos peyorativos. De este modo, cobra especial relevancia la capacidad metacognitiva de los sujetos para utilizar los elementos positivos, tanto de la motivación intrínseca como de la extrínseca, en aras de la consecución o resolución de las demandas o tareas escolares que se presenten a lo largo del curso académico.

De este modo, esta vinculación estaría en consonancia con otros trabajos que vinculan a las dimensiones del estudio. Mestre, Guil y Gil-Olarte (2004) encontraron correlaciones estadísticamente significativas entre las dimensiones de comprensión y regulación emocional junto con el rendimiento académico en un estudio donde también se atendía a la personalidad del alumnado. Vallejo et al. (2012) concluyen que el rendimiento se relaciona intrínsecamente con las tres dimensiones de la inteligencia emocional, estableciendo un vínculo asociativo entre los alumnos que obtienen una mayor puntuación de esta con el rendimiento académico y percepción de eficacia académica en la realización de tareas académicas.

Por último, Buenrostro-Guerrero et al. (2012) también atiende a la vinculación de la IE con el rendimiento académico unido a la autoeficacia y engagement académico en población adolescente. De esta manera, no se dedujo la relación de las motivaciones extrínsecas con la falta de motivación ni con las dimensiones que componen la inteligencia emocional y el rendimiento académico de una manera negativa. Por tanto, para alcanzar el éxito académico y/o un rendimiento académico óptimo, los estudiantes pueden lograr el equilibrio entre los elementos positivos que los dos tipos de motivación intrínseca y extrínseca ofrecen, abordados desde la inteligencia emocional (Couto, 2011).

Por último, la tercera hipótesis del presente estudio refería a la posible predicción positiva de las motivaciones intrínsecas y la inteligencia emocional sobre el rendimiento académico; mientras que, las motivaciones extrínsecas y amotivación lo predecirían de manera negativa. La hipótesis se cumplió de manera parcial. Se esperaba encontrar más variables predictoras; sin embargo, tan solo la motivación intrínseca hacia el conocimiento, la regulación emocional y la amotivación predijeron negativamente el rendimiento académico. Por tanto, aquellos alumnos motivados hacia el conocimiento y el deseo por el aprendizaje, quienes muestran gran capacidad para la regulación de sus estados 
emocionales y con escasos o nulos niveles de amotivación a la institución escolar predicen un rendimiento académico mayor.

La literatura científica no arroja demasiados estudios que aglutinen de manera directa los resultados hallados en esta investigación; sin embargo, muchos se aproximan de diferentes maneras. Pérez (2012), en un estudio sobre la incidencia de la inteligencia emocional en el rendimiento académico, afirma que la claridad y regulación emocional predicen negativamente la amotivación, hecho que corrobora nuestros resultados en la segunda dimensión. Recber, Isiksal y Koç (2018) niegan la influencia de la inteligencia emocional sobre el rendimiento académico en adolescentes escolares en un modelo en el que se incluyen otras variables como son la ansiedad y el estrés. Por último, Vallejo, Aja y Plaza (2018) encuentran relaciones significativas negativas entre la inteligencia emocional y el rendimiento académico mediado por otros constructos como el burnout y el estrés académico. Estos indican, además, que aquellos estudiantes con mayor nivel de rendimiento académico tienden a obtener menores niveles de inteligencia emocional y viceversa.

Por lo tanto, tal y como afirman Jiménez y López-Zafra (2009), la incidencia de la inteligencia emocional sobre el rendimiento académico de los estudiantes escolares no resulta clara ni concluyente según los resultados de las investigaciones que la literatura científica ofrece, lo que hace necesarios más estudios que indaguen en ambos constructos. Por otro lado, en referencia a las diferentes motivaciones, Cera et al. (2015) hallan la predicción negativa de la claridad emocional sobre la desmotivación del alumnado, aspecto que recalca el presente estudio, a la par las motivaciones intrínsecas y motivación extrínseca introyectada de manera positiva. A su vez, Gil-Oarte, Palomera y Brackett (2006) predicen la motivación intrínseca hacia experiencias estimulantes, el conocimiento y el logro sobre la falta de motivación del alumnado. Por su parte, Pérez (2012) predice la orientación hacia la tarea sobre la amotivación de estudiantes adolescentes.

Por todo lo enunciado, se denota la incidencia que puede llegar a tener la motivación escolar y la inteligencia emocional en el proceso socioeducativo de los estudiantes en los centros educativos. Estas, unidas a otras situaciones personales y contextuales, forman un compendio de variables psicológicas que afectan al devenir del alumno en su escolaridad, condicionando, de este modo, su rendimiento y adherencia hacia la propia institución educativa y a alcanzar un óptimo desarrollo en la vida personal y académica de los estudiantes (Bisquerra, Pérez, \& García, 2015).

\section{Limitaciones del estudio}

Las limitaciones del estudio pueden residir en su diseño transversal, tomando los datos en un momento espacio temporal dado. A su vez, los centros de educación secundaria encuestados responden de una manera aleatoria a su inclusión en el estudio, no tomando una muestra uniforme de todos los barrios o zonas de la ciudad, lo cual podría afectar, del mismo modo, a las posibles diferencias entre sus niveles socioeconómicos o educativos, así como a otras cuestiones sociales. A la par, los índices de motivación escolar, orientaciones de meta e inteligencia emocional pueden variar de un curso escolar a otro, e incluso dentro de un mismo año, en cuanto a diferentes circunstancias personales y contextuales del estudiante en su grupo/clase. 
Prospectivas de futuro

Como prospectivas de futuro, resultaría interesante llevar a cabo modelos longitudinales que permitiesen valorar la evolución de los constructos estudiados a lo largo de un período más largo de tiempo. Además, sería importante atender otras etapas educativas como pueden ser Educación Primaria o Bachillerato, de la misma manera que con otras etapas de la vida académica del estudiante como pueden ser los Grados de Formación Medio o Superior, así como la propia vida universitaria. Del mismo modo, sería relevante atender a otras variables relacionadas como pueden ser el género, la tipología de colegio, el rendimiento académico y su vinculación con las variables estudiadas, así como otros aspectos sociales y culturales.

Implicaciones prácticas

Este trabajo puede tener unas implicaciones de carácter práctico que desemboquen en unas estrategias didácticas a utilizar sobre los alumnos a través de los propios docentes u orientadores desde edades tempranas. Podrían estar orientadas hacia conductas autodeterminadas como la promoción del esfuerzo, el interés y la motivación en el estudio, que hagan a los alumnos sentirse eficaces en el desempeño de sus tareas académicas en aras de una mayor persistencia y dedicación que puedan servir como factor de prevención del bajo rendimiento académico o prematuro abandono de sus estudios.

A su vez, programas de intervención dirigidos por profesionales del ámbito en la apuesta de la Administración y/o los propios centros educativos por el trabajo con sus adolescentes en las variables acaecidas puede ayudar a contribuir a la formación integral y educacional del alumno disminuyendo el prematuro abandono escolar. Por último, los resultados de esta investigación animan a seguir investigando y buscar nuevas preguntas que ayuden a definir metodologías y a encontrar respuestas que permitan avanzar en la construcción del desarrollo socioafectivo de los adolescentes escolares.

\section{Referencias}

Abalde, E., Barca, A., Muñoz, J. M., \& Fernando, M. (2009). Rendimiento académico y enfoques de aprendizaje: una aproximación a la realidad de la enseñanza superior brasileña en la región norte. Revista de Investigación Educativa, 27(2), 303-3019.

Alemany, I., Campoy, I., Ortiz, M., \& Benzaquén, R. (2015). Las orientaciones de meta en el alumnado de secundaria: Un análisis en un contexto multicultural. Publicaciones, $45,83-100$.

Alexandris, K. (2013). Segmenting recreational tennis players according to their involvement level: A psychographic profile based on constraints and motivation. Managing Leisure, 18(3), 179-193. doi: 10.1080/13606719.2013.796178

Anaya, D. (2012). Bases del aprendizaje y educación. Madrid: Sanz y Torres.

Andaur, A. \& Berger, C. (2018). Implementación e impacto de un taller de autocuidado basado en mindfulness en profesionales de la educación. Estudios sobre Educación, $34,239-261$.

Actualidades en Psicología, 32(125), 2018, 95-112 
Anderman, E., Anderman, L., Yough, M., \& Gimbert, B. (2010). Value-added models of assessment: Implications for motivacion and accountability. Educational Psychologist, 45(2), 123-137.

Asociación Médica Mundial (AMM). (2000). Declaración de Helsinki. Principios éticos para las investigaciones con los seres humanos. Seúl, (Corea.): Autor.

Barca, A., Peralbo, M., Porto, Marcos, J., \& Brenlla, J. (2011). Metas académicas del alumnado de Educación Secundaria Obligatoria (ESO) y Bachillerato con alto y bajo rendimiento escolar. Revista de Educación, 354, 341-368.

Bisquerra, R., Pérez, J. C., \& García, E. (2015). Inteligencia emocional en educación. Madrid: Síntesis.

Bresó, E., Schaufeli, W. B., \& Salanova, M. (2011). Can a self-efficacy-based intervention decrease burnout, increase engagement, and enhance performance? A quasiexperimental study. Higher Education, 61(4), 339-355. doi: 10.1007/s10734-010-9334-6

Buenrostro-Guerrero, A. E., Valadez-Sierra, M. D., Soltero-Avelar, R., Nava-Bustos, G., Zambrano-Guzmán, R., \&y García-García, A. (2012). Inteligencia emocional y rendimiento académico en adolescentes. Revista de Educación y Desarrollo, 20, 29-37.

Carmona, C., Sánchez, P., \& Bakieva, M. (2011). Actividades extraescolares y rendimiento académico: diferencias en autoconcepto y género. Revista de Investigación Educativa, 29(2), 447-465.

Cera, E., Almagro, B., Conde, C., \& Sáenz-López, P. (2015). Inteligencia emocional y motivación en educación física en secundaria. Retos. Nuevas Tendencias en Educación Física, Deporte y Recreación, 27, 8-13.

Córdoba, L., García, V., Luengo, L., Vizuete, M., \& Feu, S. (2012). How academic career and habits related to the school environment influence on academic performance in the physical education subject. Retos. Nuevas tendencias en Educación Física, Deporte y Recreación, 21, 9-13.

Couto, S. (2011). Desarrollo de la relación entre inteligencia emocional y los problemas de convivencia: estudio clínico y experimental. Madrid: Visión libros.

Deci, E. L., \& Ryan, R. M. (2002). Self-determination research: Reflections and future directions. In E.L. Deci y R.M. Ryan (Eds.), Handbook of self-determination research. (pp. 431-441). University of Rochester Press, Rochester (UK).

Delgado, B.; Inglés, C.,; García-Fernández, J.; Castejón, J., \&Valle, A. (2010). Diferencias de género y curso en alumnos de Educación Secundaria Obligatoria. Revista Española de Pedagogía, 68(245), 67-84.

Extremera, N., Durán, A., \& Rey, L. (2009). The moderating effect of trait meta-mood and perceived stress on life satisfaction. Personality and Individual Differences, 47(2), 116-121. doi: 10.1016/j.paid.2009.02.007

Fernández-Berrocal, P., Extremera, N., \& Ramos, N. (2004). Validity and reliability of the Spanish modified version of the Trait Meta-Mood Scale. Psychological Reports, 94(3), 751-755. 
Ferriz, R., Sicilia, A., \& Sáenz, P. (2013). Predicting satisfaction in physical education classes: A study based on self determination theory. The Open Education Journal, 6, 1-7.

Gaeta, M. \& Cavazos, J. (2015). Implicación académica en matemáticas: percepción de metas docentes y procesos autorregulatorios en estudiantes de Bachillerato. Revista Ibero-americana de Estudos em Educação. (4), 951-968. doi: 10.1016/S01200534(15)30002-9

Garzón, R., Rojas, M. O., Del Riesgo, L., Pinzón, M., \& Salamanca, A. L. (2010). Factores que pueden influir en el rendimiento académico de estudiantes de Bioquímica que ingresan en el programa de Medicina de la Universidad del Rosario-(Colombia). Educación Médica, 13(2), 85-96.

Goleman, D. (2012). Inteligencia emocional. España: Editorial Kairós S. A.

Gómez-Fraguela, J. A., Fernández, N., Romero, E., \& Luengo, A. (2008). El botellón y el consumo de alcohol y otras drogas en la juventud. Trastornos Adictivos, 20(2), 211-217.

Hernando, A., Oliva, A., \& Pertegal, M. (2012). Variables familiares y rendimiento académico en la adolescencia. Estudios de Psicología, 33(1), 51-65. doi: 10.1174/021093912799803791

Järvenoja, H. (2010). Socially shared regulation of motivation and emotions in collaborative learning. Acta Universitatis Ouluensis E Scientiae Perum Socialium, 110. Recuperado de: http://herkules.oulu.fi/isbn9789514263309/isbn9789514263309.pdf

Jiménez, I., \& López-Zafra, E. (2009). Inteligencia emocional y rendimiento escolar: estado actual de la cuestión. Revista Latinoamericana de Psicología, 41(1), 69-79. doi: 10.14349/rlp.v41i1.556

Mayer, J. D., Roberts, R. D., \& Barsade, S. G. (2008). Human abilities: Emotional intelligence. Annual Review of Psychology, 59(1), 507-536. doi: 10.1146/ annurev. psych.59.103006.093646

Mayer, J. D., \& Salovey, P. (1997). What is emotional intelligence? En P. Salovey \& D. Sluyter (Eds.), Emotional development and emotional intelligence: Educational applications (pp. 3-31). New York: Basic Books.

Mayer, J. D., Salovey, P., \& Caruso, D. R. (2000). Models of emotional intelligence. En R. J. Sternberg (Ed.), Handbook of intelligence (pp. 396-420). Cambridge: Cambridge University Press.

Mestre, J., Guil, M., \& Gil-Olarte, P. (2004). Inteligencia emocional: Algunas respuestas empíricas y su papel en la adaptación escolar en una muestra de alumnos de secundaria. Revista Electrónica de Motivación y Emoción, 6(16), 2.

Molleda, C. B. \& Herrero, F. J. (2009). Responsabilidad y comportamiento antisocial del adolescente como factores asociados al rendimiento escolar. Acta colombiana de psicología, 12(2), 69-76.

Montero, I., \& León, O. (2007). A guide for naming research studies in Psychology. International Journal of Clinical and Health Psychology, 7, 847-862. 
Moreno, J. \& Martínez, A. (2006). Importancia de la teoría de la autodeterminación en la práctica físico-deportiva: fundamentos e implicaciones prácticas. Cuadernos de Psicología del Deporte. 6 (2). 39-54.

Musitu, G., Jiménez, T., \& Murgui, S. (2012). Funcionamiento familiar, autoestima y consumo de sustancias en adolescentes: un modelo de mediación. Revista de salud pública de México, 49(1), 3-10.

Navas, L. \& Sampascual, G. (2008). Un análisis exploratorio y predictivo sobre las orientaciones de meta sobre el contenido de las metas de los estudiantes. Horizontes Educacionales, 13(1), 23-34.

Niebla, J. \& Hernández, L. (2007). Variables que inciden en el rendimiento académico de adolescentes mexicanos. Revista latinoamericana de psicología, 39(3), 487-501.

Núñez, J. (2009). Motivación, aprendizaje y rendimiento académico. Actas do X Congresso Internacional Galego-Português de Psicopedagogia. Braga: Universida de do Minho. Recuperado de: http://www.educacion.udc.es/grupos/gipdae/ documentos/congreso/Xcongreso/pdfs/cc/cc3.pdf

Núñez, J., Martín-Albo, J., Navarro, J., \& Suárez, Z. (2010). Adaptación y validación de la versión española de la Escala de Motivación Educativa en estudiantes de educación secundaria postobligatoria. Estudios de Psicología, 31(1), 89-100.

Palacio, S., Caballero, C., González, O., Gravini, M., \& Contreras, K. (2012). Relación del burnout y las estrategias de afrontamiento con el rendimiento académico en estudiantes universitarios. Universytas Psychological, 11(2), 234-239.

Pena, M., Rey, L., \& Extremera, N. (2012). Life satisfaction and engagement in elementary and primary educators: Differences in emotional intelligence and gender. Revista de Psicodidáctica, 17(2), 341-358. doi: 10.1387/Rev.Psicodidact.1220

Pérez, A. (2012). Inteligencia emocional y motivación del estudiante universitario. (Tesis Doctoral). ULPGC: Las Palmas de Gran Canaria.

Pérez, N. \& Castejón, J. (2006). Relaciones entre la inteligencia emocional y el cociente intelectual con el rendimiento académico en estudiantes universitarios. Revista Electrónica de Motivación y Emoción, 9(22). Universidad de Alicante .

Pintrich, P. R. \& Schunk, D. H. (2006). Motivación en contextos educativos. Teoría, investigación y aplicaciones. Madrid: Pearson Educación.

Recber, S., Isiksal, M. \& Koç, Y. (2018). Investigating self-efficacy, anxiety, attitudes and mathematics achievement regarding gender and school type. Anales de psicología, 34(1), 41-51.

Saies, E., Arribas, S., Cecchini, J. A., De-Cos, I. \& Otaegi, O. (2014). Diferencias en orientación de meta, motivación autodeterminada, inteligencia emocional y satisfacción con los resultados deportivos entre piragüistas expertos y novatos. Cuadernos de Psicología del Deporte, 14(3), 21-30. 
Salavera, C. \& Usán, P. (2018). Adaptación del cuestionario de competencia interpersonal ICQ-15 con población adolescente hispanohablante. Revista iberoamericana de diagnóstico y educación. doi: 10.21865/RIDEP48.3.03

Salavera, C., Usán, P. \& Jarie, L. (2017). Emotional intelligence and social skills on selfefficacy in Secondary Education Students. Are there gender differences? Journal of Adolescence, 60, 39-46. doi: 10.1016/j.adolescence.2017.07.009

Salavera, C., Usán, P., \& Jarie, L. (2018). Styles of humor and social skills in students. Gender differences. Current Psychology. doi: 10.1007/s12144-017-9770-x

Salavera, C., Usán, P. Jarie, L., \& Lucha, O. (2018). Sentido del humor, afectos y personalidad. Estudio en estudiantes universitarios. Avances en psicología latinoamericana, 36(1), 8391. doi: 10.12804/revistas.urosario.edu.co/apl/a.4511

Salovey, P., Mayer, J. D., Goldman, S. L., Turvey, C., \& Palfai, T. P. (1995). Emotional attention, clarity and repair: exploring emotional intelligence using the Trait Meta-Mood Scale. En J.W. Pennebaker (Ed.), Emotion, disclosure and health, 125154. Washington: American Psychological Association. doi: 10.1037/10182-006

Sanz, M., Menéndez, F., Rivero, M. y Conde M. (2009). Psicología de la motivación. Madrid: Editorial Sanz y Torres.

Skinner, E., Furrer, C., Marchand, G., \& Kinderman, T. (2008). Engagement and disaffection in the classroom: Part of a larger motivational dynamic? Journal of Educational Psychology, 100(4), 765-781.

Tilano, L. M., Henao, G., \& Restrepo, J. (2009). Prácticas educativas familiares y desempeño académico en adolescentes escolarizados en el grado noveno de instituciones educativas oficiales del municipio de Envigado. El Ágora USB, 9(1), 35-51. doi: $10.21500 / 16578031.404$

Tsouloupas C., Carson, R., Matthews, R., Grawitch, M., \& Barber, L. (2010). Exploring the association between teachers' perceived student misbehaviour and emotional exhaustion: The importance of teacher efficacy beliefs and emotion regulation. Educational Psychology, 30(2), 173-189. doi: $10.1080 / 01443410903494460$

Usán, P. \& Salavera, C. (2017). Influencia de la motivación hacia el deporte en el consumo de alcohol, tabaco y cannabis de adolescentes escolares. Actualidades en Psicología, 31(122), 119-131. doi: 10.15517/ap.v31i122.27980

Usán, P., Salavera, C. \& Domper, E. (2018). ¿Cómo se interrelacionan las variables de burnout, engagement y autoeficacia académica? Un estudio con adolescentes escolares. Revista Electrónica Interuniversitaria de Formación del Profesorado, 21(2), 141 153. doi: $10.6018 /$ reifop.21.2.311361

Usán, P., Salavera, C., Merino, A., \& Jarie, L. (2018). Satisfacción de necesidades psicológicas y orientaciones de meta en profesorado de Educación Física hacia sus alumnos. Retos. Nuevas tendencias en educación física, deporte y recreación, 33(1), 50-53. 
Vallejo, B., Martínez, J., García, C., \& Rodríguez, C. (2012). Influencia de la inteligencia emocional en el rendimiento escolar. Comunicación científica. XIII Congreso virtual de psiquiatría.

Vallejo, M., Aja, J., \& Plaza, J. J. (2018). Estrés percibido en estudiantes universitarios: Influencia del burnout y del engagement académico. International Journal of eductional research and innovation, 9, 220-236.

Zimmerman, B. J. (2008). Goal setting: A key proactive source of academic self-regulation. En B. J. Zimmerman \& D. H. Schunk (Eds.). Motivation and self-regulated learning: Theory, research, and applications (267-295). Mahwah, N. J. US: Lawrence Erlbaum Associates Publishers

Recibido: 15 de febrero, 2018

Aceptado: 09 de agosto, 2018 